\title{
WORKSHOP
}

\section{DRAFT OF A SYSTEM FOR SOLVENCY CONTROL IN NON-LIFE INSURANCE*}

\author{
By Ragnar Norberg and BJørn Sundt \\ Institute of Mathematics, University of Oslo
}

\begin{abstract}
An outline is given of a proposed system for solvency control in non-life insurance that has recently been discussed within a Working Party appointed by the Norwegian supervisory authorities. According to this system the factual technical reserves must at any time be sufficient to meet, with high probability, all future liabilities stipulated by insurance contracts that have either expired or are currently in force. The system is applied to a provisional, simple model that has been fitted to claims data assembled from Norwegian non-life companies. The numerical examples illustrate, inter alia, how the required reserve depends on the volume of the business, the portfolio mix, and the reinsurance cover.
\end{abstract}

\section{INTRODUCTION}

In 1978 a commission was appointed by the Norwegian government to work out a proposal for a new insurance company act. One central task in the commission's mandate, apparently urged by some recent instances of failure of non-life insurance companies, was to work out regulations for an improved governmental supervision of non-life insurance enterprises. The commission presented its report (HARLem et al. (1983)) at the end of 1983.

Envisaging the preparation of a strengthened legislation, the Norwegian supervisory authorities in March 1982 appointed a Working Party on technical aspects of supervision in non-life insurance, with members-most of them actuariesfrom the non-life insurance industry, the University of Oslo, and the Supervisory Service.

A central item in the mandate of the Working Party was to propose regulations for the determination of technical reserves required in non-life insurance and for the reporting of statistical data from the non-life companies to the supervisory authorities. In summary, the task of the Working Party was to propose a system for solvency control of the non-life insurance business. The Working Party presented its report (JoHNSEN et al. (1984)) in May 1984.

The authors of the present paper were involved in this project; one was a member of the Working Party, and the other was engaged by the Supervisory Service to assist the Working party with statistical analyses. In the present report we offer an outline of a system for solvency control based on the discussions in

* A preliminary version of the present paper was presented at the 17th ASTIN Colloquium, Lindau, October 1983. Parts of the present research were performed while the second author was staying at the Norwegian Insurance Supervisory Service, the University of Mannheim, and the University of Copenhagen. 
the Working Party. The ideas put forward here must be read as representing a purely personal judgement which is not in perfect agreement with the recommendations of the Working Party.

\section{AN OUTLINE OF THE SOLVENCY CONTROL SYSTEM; BASIC PRINCIPLES}

2A. Definitions. We consider a non-life insurance company and introduce the following notation related to the business net of reinsurance:

$X_{j, d}$ is the total amount paid in year $j+d$ in regard of claims occurred in year $j$ (i.e., with a delay of $d$ years);

$\dot{X}_{j, d}$ is the total amount paid in year $j+d$ in regard of claims occurred in year $j$ for which the company had assumed liability at the beginning of year $j$.

The amount $\dot{X}_{j, d}$ is clearly contained in $X_{j, d .}$. Whereas $\dot{X}_{j, d}$ comprises only payments in respect of claims occurred in year $j$ for which the company was liable already at the beginning of year $j, X_{j, d}$ also includes payments on claims occurred in year $j$ for which the company has assumed liability during that year.

Suppose a solvency analysis is to be carried out for the company at time $J$ (the beginning of year $J$ ). A key quantity is

$X$, the total amount of future claim payments for which the company has assumed liability at time $J$.

We assume that at time $J$ the company has assumed no liability for claims occurring after year $J$, and thus $X$ is made up of

$$
\begin{gathered}
\dot{X}_{J .}=\sum_{d=0}^{\infty} \dot{X}_{J, d}, \begin{array}{l}
\text { future payments in regard of claims occurring in year } J \text { for } \\
\text { which the company has assumed liability by the } \\
\text { beginning of year } J,
\end{array}
\end{gathered}
$$

and

$$
R_{J}=\sum_{j, d ; j<J \leqslant j+d} X_{j, d}, \begin{aligned}
& \text { future payments in regard of claims occurred previous } \\
& \text { to year } J ;
\end{aligned}
$$

hence

$$
X=\dot{X}_{J}+R_{J} .
$$

(If desired, a discount factor could be introduced in the expressions defining $\dot{X}_{J}$. and $R_{J}$.)

When a stochastic model for the risk process is introduced, $X$ is represented as a random variable. The distribution of $X$ may depend on the past claims history as summarized in the claims statistics available by time $J$, which we denote by $S_{J}$. In particular, $R_{J}$ may be significantly depending on $S_{J}$ due to interdependence between the amounts $X_{j, d}$ paid in different development years $d$ for a fixed year of origin, $j$. Thus, the relevant distribution to employ for an assessment of 
the total risk carried by the company at time $J$, is the conditional distribution of $X$ given $S_{J}$.

2B. Solvency Requirements. By time $J$ the company has already received from the insurants the premiums for the coverage of $X$. Therefore a technical reserve $u$ has to be set aside to defray these expences, and it must be determined with a view to the random nature of the latter. The solvency regulations stipulate:

(I) The net technical reserve $u$ must be sufficiently large to cover $X$ with a probability not less than $1-\varepsilon$, that is,

$$
P\left(X \leqslant u \mid S_{J}\right) \geqslant 1-\varepsilon .
$$

Defining the minimum reserve $u_{\min }$ as the smallest $u$ satisfying (1), we recast the requirement (1) as

$$
u \geqslant u_{\min }
$$

which is suitable for the purpose of solvency control. (By definition, $u_{\min }$ is an upper $\varepsilon$-fractile of the conditional distribution of $X$ given $S_{J}$.)

It is customary to decompose the technical reserve into a premium reserve, a loss reserve, and (possibly) a fluctuation reserve. As theoretical counterparts of these accounting concepts we introduce the premium liability

the loss liability

$$
u_{p}=E\left(\dot{X}_{J} \mid S_{J}\right) \text {, }
$$

$$
u_{l}=E\left(R_{J} \mid S_{J}\right) \text {, }
$$

and finally, the fluctuation liability $u_{f}$ determined by the equation

$$
u_{\min }=u_{p}+u_{l}+u_{f}
$$

In regard of relation (3) we could alternatively speak of $u_{\min }$ as the total liability. It is only $u_{\min }$ that appears in the solvency requirement (2). The auxiliary terms appearing on the right hand side of (3) serve to explain how the total liability is composed. The first two terms show how the expected payments divide in two components, one in regard of future claims covered by policies that are currently in force, and the other in regard of claims that have already occurred. The last term is a kind of safety loading.

In conjunction with the requirement (I) the regulations must also lay down specific requirements as to the security of the technical reserves, hence:

(II) The supervisory authorities establish rules as to which assets can be accounted as corresponding to technical reserves. In principle only investments judged to be safe can be approved of as corresponding to technical reserves.

As it stands, requirement (II) may appear rather severe, and an amplification is called for. The intention is, of course, not that only risk-free assets can be approved of, but rather that the valuation of these assets should be sufficiently prudent to ensure their adequacy to cover the technical reserves in case of a forced sale brought about by a possible failure and closing of the company to 
new business. An alternative approach would be to extend the stochastic model framework so as to include also a description of the uncertainty associated with the investment portfolio. It is, however, felt that a formal model would not provide a more reliable valuation of assets than that obtained by a skillful subjective assessment; hence we remain with the present formulation of requirement (II). The point of the theory will become clear when item (II) is read in conjunction with item (IV) below.

To check whether the solvency requirements stipulated above are satisfied, there must be a regular solvency control:

(III) The Supervisory Service verifies regularly (e.g., annually) that the technical reserves are secured in accordance with (I) and (II).

Requirements (1)-(III) deal with the maintenance of the technical reserves in companies that are in a solvent state. However, the very purpose of the technical reserve is to ensure that legal insurance claims can be indemnified even if the company goes bankrupt. Therefore, the legislation must authorize that:

(IV) In case of realization of the assets corresponding to technical reserves, insurance claims rank prior to all other claims.

The statutory solution could basically be borrowed from life insurance. Even though non-life insurance and life insurance are fundamentally different in respect of the duration of the contracts, they are comparable as regards their capacity to create a permanent reserve. In fact, for a company with a constant size and portfolio mix, the minimum reserve determined by (1) remains unchanged over time.

A leading principle that can be derived from items (I)-(IV), is the separation of the insurance business from the other economic activities of the company. The solvency control system outlined here is concerned solely with the insurance business and sees that it solves its basic task as a social security system. Losses suffered by an insurance company in connection with its non-insurance activities will not hit the insurants, even if the company has to be wound up.

\section{STATISTICAL PROCEDURES AND REQUIREMENTS}

It is sometimes argued that a refined statistical analysis of claims data is of limited importance since the risk associated with the asset portfolio is more critical (actually the most recent instance of insolvency in Norwegian non-life insurance was caused by failure of general business). This point of view pays regard only to the pure business goals of the company. We stress once more that the necessity of measuring the insurance risk accurately arises from the needs of the insurants; the technical reserves represent a guarantee that their insurance claims will be covered (with high probability). A reliable determination of $u_{\min }$ is, therefore, an essential part of the solvency control. (Besides, the present authors do not share the opinion that the risk associated with the insurance coverages is of subordinate importance; history provides ample evidence to the contrary.) 
The possibility of providing supervisory authorities with objective and scientifically based methods for determination of adequate technical reserves was discussed in the Working Party. In this connection a pilot study was carried through, in which statistical data were assembled from a number of Norwegian non-life companies. The discussions and the experience obtained from the study uncovered a multitude of problems that have to be settled before a statistically based system of solvency control can be set in full operation. The discussion below will indicate some points.

The organization of the statistics files differs from one company to another, and even the definitions of basic concepts are not always universal. Therefore the supervisory authorities must, in cooperation with the insurance companies, work out standards for definitions and organization of data bases to make them compatible with a central statistically based system of solvency control.

In many companies claims statistics are produced with a primary view to accounting rather than statistical analysis. Consequently, the data available to the Supervisory Service are often too aggregate to allow for an identification of the technical results in risk classes of special importance, viz. those in rapid growth and those related to new kinds of coverage. Furthermore, the claims data are usually not related to the information in the policy files and, consequently, a risk analysis cannot be accomplished.

To put the latter remark more precisely, we make some simple model assumptions. The portfolio is composed of $I$ risk classes, numbered from 1 to $I$. Each class is homogeneous with respect to basic risk characteristics, that is, the individual risks in one and the same class have identical values of certain basic tariff entries. It is assumed that each risk class generates claims in accordance with a compound Poisson process. Thus, letting $p_{i ; j}$ and $X_{i ; j}$ denote, respectively, the volume of risk exposed and the total amount of claims in class $i$ and year $j$, we assume that $X_{i ; j}$ follows a compound Poisson probability law with frequency parameter $p_{i ; j} \lambda_{i}$ and claim size distribution $G_{i}$. In shorthand we write this as

$$
X_{i ; j} \sim \operatorname{comp.~Po.~}\left(p_{i ; j} \lambda_{i}, G_{i}\right)
$$

The risk exposure $p_{i ; j}$ can be the number of risks, the number of risk years, the total of sums insured, the total mileage (in automobile insurance), or some other physical/technical measure of the "risk volume" of class $i$ in year $j$. It is clearly unsatisfactory to use the premium income as such a measure since it, roughly speaking, is a product of volume and price, the latter being subject to variations in market conditions and partly controlled by the insurer. By a tariff revision the premium income will be increased, say, whereas the risk will remain unchanged. (When comparing distances we should apply a common yard-stick and not switch from meters to yards.) The parameter $\lambda_{i}$ can be interpreted as the intensity, with respect to operational time, of the underlying compound Poisson process.

The risk classes are grouped into $H(H \leqslant I)$ statistics branches $B_{1}, \ldots, B_{H}$, say, which form the basis for the regular reporting of statistics from the companies to the Supervisory Service. 
The total amount of claims in branch $B_{h}$ in year $j$ is

$$
X_{h j}=\sum_{i \in B_{h}} X_{i ; j}
$$

Under the assumptions specified above, we have that

where

$$
X_{h j} \sim \text { comp. Po. }\left(\lambda_{h j}, G_{h j}\right),
$$

$$
\lambda_{h j}=\sum_{i \in B_{h}} p_{i ; j} \lambda_{i}
$$

and

$$
G_{h j}=\frac{1}{\lambda_{h j}} \sum_{i \in B_{h}} p_{i ; j} \lambda_{i} G_{i}
$$

In its annual report for year $j$ to the Supervisory Service the company provides, for each branch $B_{h}$, the number of claims, $N_{h j}$, and the empirical distribution of the single claim amounts $Y_{h j k}, k=1, \ldots, N_{h j}$. We have that

$$
N_{h j} \sim \text { Po. }\left(\lambda_{h j}\right)
$$

and

$$
Y_{h j k}, k=1,2, \ldots \text { are i.i.d. } \sim G_{h j},
$$

where Po. $(\lambda)$ denotes the Poisson distribution with parameter $\lambda$, and "i.i.d." means "independent and identically distributed". From these statistics the Supervisory Service is able to estimate the branch parameters $\lambda_{h j}$ and $G_{h j}$ given by (4) and (5), and only these.

We are now in a position to draw some conclusions as to what information is needed to estimate the distribution of the next year's total claim amount.

Let us first assume that the annual report for branch $B_{h}$ gives complete information about the number of claims $N_{h j}$ and the single claim amounts $Y_{h j k}$, but that the risk exposures are completely unknown. From the statistics from the past years $1, \ldots, J^{*}$ one can estimate the parameters $\lambda_{h j}$ and $G_{h j}, j=1, \ldots, J^{*}$, and only these. It is clear that for an aggregate branch the future $\left(\lambda_{h J}, G_{h J}\right)$ can be estimated from the available statistics if and only if $\left(\lambda_{h j}, G_{h j}\right)=\left(\lambda_{h J}, G_{h J}\right)$ for some former years, say $j=1, \ldots, j^{*}$. This relation holds true for all values of $\lambda_{i}$ and $G_{i}, i \in B_{h}$, if and only if $p_{i, j}=p_{i ; J}$ for all $j=1, \ldots, J^{*}$ and $i \in B_{h}$. We conclude that if the single class exposures $p_{i, j}$ are not observed, then the distribution of the next year's total claim amount is estimable if and only if the branch $B_{h}$ has constant size and composition.

Now assume that the annual report in addition to $N_{h j}$ and the $Y_{h j k}$ 's also contains the value of the total exposure

$$
p_{h j}=\sum_{i \in B_{h}} p_{i j j}
$$

of branch $B_{h}$ in year $j$. Then the above conclusion will still hold unless we make further assumptions about the $p_{i, j}$ 's. Assume, for instance, that the composition 
of the branch is constant, that is, there exist (unknown) constants $a_{i}, i \in B_{h}$ such that

$$
p_{i ; j}=a_{i} p_{h j}, \quad j=1, \ldots, J^{*}, J ; \quad i \in B_{h} .
$$

Then (4) and (5) reduce to

$$
\lambda_{h j}=p_{h j} \lambda_{h}
$$

and

where

$$
G_{h j}=G_{h .}
$$

$$
\lambda_{h .}=\sum_{i \in B_{h}} a_{i} \lambda_{i}
$$

and

$$
G_{h .}=\frac{1}{\lambda_{h .}} \sum_{i \in B_{h}} a_{i} \lambda_{i} G_{i}
$$

In this case $\lambda_{h J}$ and $G_{h J}$ can be estimated from the available data. Note that the claim size distribution $G_{h J}$ can be estimated even without knowledge of the values of the $p_{h j}$ 's as long as the composition of the branch is constant.

In the special case where the branch is homogeneous (i.e., consists of only one risk class), the composition of the branch is trivially constant, and thus the total claim distribution of the next year can be estimated if the risk exposures are observed.

With the above theoretical discussion in mind we formulate the following general requirements (V) and (VI) concerning the regular reporting and the internal recording of statistics:

(V) The supervisory authorities must design statement forms with entries for the number of claims and the empirical claim size distribution as per year of occurrence and development, and premium income as well as appropriate physical/technical measures of risk exposure for each branch. All quantities are to be specified for the gross business, and the reinsurance programme is to be described. The subdivision into statistics branches should be sufficiently refined so that each branch is fairly homogeneous with respect to significant risk characteristics.

In special cases it may be necessary to call for supplementary information. In principle, the Norwegian Supervisory Service is already by the present statutory regulations entitled to require any statistical information necessary to accomplish a reliable solvency control. A basic element of a system for solvency control is the following general requirement:

(VI) The internal data bases of the companies must be organized in a manner that allows for a detailed risk analysis. This implies that complete information on risk characteristics and claims experience must be recorded for each single risk. 


\section{THE MINIMUM RESERVE}

By definition, the minimum reserve $u_{\min }$ is the upper $\varepsilon$-fractile of the distribution of $X$ (strictly speaking, of the conditional distribution of $X$ given $S_{\mathrm{J}}$, but for simplicity we leave aside the explicit mentioning of the conditioning in the following). For the determination of $u_{\min }$ we use the NP-approximation,

$$
u_{\min } \approx E X+c_{1} \sqrt{\operatorname{Var} X}+c_{2} E(X-E X)^{3} / \operatorname{Var} X,
$$

where $c_{1}$ is the upper $\varepsilon$-fractile of the standard normal distribution $N(0,1)$ and $c_{2}=\left(c_{1}^{2}-1\right) / 6$ [cf. Beard, Pentikäinen and Pesonen (1984)].

We assume that the insurance company works in $H$ different insurance branches, and that

(i) events referring to different branches are stochastically independent.

Let $X_{h}$ be the part of $X$ originating from branch $h$. Then we have

$$
X=\sum_{h=1}^{H} X_{h}
$$

and, by assumption (i),

$$
\begin{gathered}
E X=\sum_{h=1}^{H} E X_{h}, \\
\operatorname{Var} X=\sum_{h=1}^{H} \operatorname{Var} X_{h}, \\
E(X-E X)^{3}=\sum_{h=1}^{H} E\left(X_{h}-E X_{h}\right)^{3} .
\end{gathered}
$$

Our next step is to model the total claim amounts $X_{h}$. We make the following assumptions (ii) and (iii) about the occurrence, size, and regulation of the claims.

(ii) All claims are settled immediately when they occur.

This assumption is of course unrealistic, and we shall adjust for it later (cf. subsection 6A). However, it makes the following much simpler. Assumption (ii) implies in particular that $R_{J}=0, X=\dot{X}_{J .}=\dot{X}_{J, 0}$, and $u_{l}=0$.

Let $\dot{N}_{h J}$ be the number of claims included in $X_{h}$, and let $\dot{Y}_{h J k}$ be the net amount (after reinsurance) of the $k$ th of these claims. Thus we have

$$
X_{h}=\sum_{k=1}^{N_{h J}} \dot{Y}_{h J k}
$$

with the convention that $X_{h}=0$ when $\dot{N}_{h J}=0$. The introduction of variables like the $\dot{Y}_{h J k}$ 's implies that we exclude reinsurance forms like stop-loss, ECOMOR, largest claims, etc. 
We make the following distributional assumptions:

(iii) The number of claims $\dot{N}_{h J}$ is conditionally Poisson distributed with parameter $\dot{p}_{h J} \theta$, given the value $\theta$ of an unknown random parameter $\Theta_{h J}$. The net claim amounts $\dot{Y}_{h{ }_{k}} ; k=1,2, \ldots$ are i.i.d. and independent of $\dot{N}_{h J}$ and $\Theta_{h J}$.

The quantity $\dot{p}_{h J}$ appearing in assumption (iii) is a measure of risk exposure, cf. Section 3. The reason for modelling the Poisson parameter as a random variable, is that there are collective unpredictable factors influencing the whole portfolio; a cold winter with icy roads may cause many car accidents, etc. It may be an unrealistic assumption that the collective risk factors influence only the claim frequencies, not the claim amounts; icy roads may cause a special kind of accidents.

We shall have to make some additional assumptions in Section 5 where we deal with estimation of the model parameters.

We introduce the parameters

$$
\left\{\begin{array}{l}
\dot{\alpha}_{i h J}=E\left(\dot{Y}_{h J 1}\right)^{i}, \quad i=1,2,3, \\
\beta_{1 h}=E \Theta_{h J}, \\
\beta_{2 h}=\operatorname{Var} \Theta_{h J}, \\
\beta_{3 h}=E\left(\Theta_{h J}-E \Theta_{h J}\right)^{3},
\end{array}\right.
$$

and we easily get [cf. e.g. RANTALA (1982)]

$$
\begin{gathered}
E X_{h}=\dot{p}_{h J} \beta_{1 h} \dot{\alpha}_{1 h J}, \\
\operatorname{Var} X_{h}=\dot{p}_{h J} \beta_{1 h} \dot{\alpha}_{2 h J}+\left(\dot{p}_{h J}\right)^{2} \beta_{2 h}\left(\dot{\alpha}_{1 h J}\right)^{2}, \\
E\left(X_{h}-E X_{h}\right)^{3}=\dot{p}_{h J} \beta_{1 h} \dot{\alpha}_{3 h J}+3\left(\dot{p}_{h J}\right)^{2} \beta_{2 h} \dot{\alpha}_{1 h J} \dot{\alpha}_{2 h J}+\left(\dot{p}_{h J}\right)^{3} \beta_{3 h}\left(\dot{\alpha}_{1 h J}\right)^{3} .
\end{gathered}
$$

In practical computations of the moments (11)-(13), the risk volumes $\dot{p}_{h J}$ would depend on the insurance company, whereas the estimates of the other parameters are supposed to be supplied by the Supervisory Service. For computational ease, we introduce the following reparametrization of (11)-(13):

$$
\begin{gathered}
E X_{h}=\dot{p}_{h J} \dot{\mu}_{1 h J}, \\
\operatorname{Var} X_{h}=\left(\dot{\mu}_{2 h J}+\rho_{h} E X_{h}\right) E X_{h}, \\
E\left(X_{h}-E X_{h}\right)^{3}=\dot{\mu}_{3 h J} E X_{h}+3 \dot{\mu}_{2 h J} \operatorname{Var} X_{h}+\tau_{h} E^{3} X_{h},
\end{gathered}
$$

with

$$
\begin{aligned}
\dot{\mu}_{1 h J} & =\beta_{1 h} \dot{\alpha}_{1 h J}, \\
\dot{\mu}_{2 h J} & =\dot{\alpha}_{2 h J} / \dot{\alpha}_{1 h J}, \\
\dot{\mu}_{3 h J} & =\left(\dot{\alpha}_{3 h J} / \dot{\alpha}_{1 h J}\right)-3\left(\dot{\mu}_{2 h J}\right)^{2}, \\
\rho_{h} & =\beta_{2 h} /\left(\beta_{1 h}\right)^{2}, \\
\tau_{h} & =\beta_{3 h} /\left(\beta_{1 h}\right)^{3} .
\end{aligned}
$$


In practice it could often be difficult to find good estimates for $\beta_{2 h}$ and, in particular, $\beta_{3 h}$, and it could therefore be necessary to give more subjective estimates of $\rho_{h}$ and $\tau_{h}$. For this purpose, it could be practical to express these parameters by the coefficient of variation $\kappa_{2 h}=\beta_{2 h}^{1 / 2} / \beta_{1 h}$ and the skewness $\kappa_{3 h}=\beta_{3 h} /\left(\beta_{2 h}\right)^{3 / 2}$ of the distribution of $\theta_{h J}$ as it is easier to have an intuitive opinion about these than about $\beta_{2 h}$ and $\beta_{3 h}$. We have

$$
\begin{aligned}
& \rho_{h}=\left(\kappa_{2 h}\right)^{2}, \\
& \tau_{h}=\kappa_{3 h}\left(\kappa_{2 h}\right)^{3} .
\end{aligned}
$$

The parameters $\dot{\alpha}_{i h J}$ (and $\dot{\mu}_{i h J}$ ) depend on the reinsurance cover, whereas it is natural to assume that the $\beta_{i h}$ 's are independent of the reinsurance cover. Therefore, the Supervisory Service would have to provide the company with tables of the $\dot{\alpha}_{i h J}$ 's (or $\dot{\mu}_{i h J}$ 's) for different reinsurance forms and different retentions. In the following we discuss the reinsurance forms quota-share, excess-of-loss, and surplus.

Let $\dot{Z}_{h J k}$ be the gross amount (before reinsurance) of the $k$-th claim contained in $X_{h}$. For quota-share with retention $q$ we clearly have

$$
\dot{Y}_{h J k}=q \dot{Z}_{h J k}
$$

and thus

$$
\begin{aligned}
\dot{\alpha}_{i h J}(q) & =q^{i} \dot{\alpha}_{i h J}(1), \quad i=1,2,3, \\
\dot{\mu}_{1 h J}(q) & =q \dot{\mu}_{1 h J}(1), \\
\dot{\mu}_{2 h J}(q) & =q \dot{\mu}_{2 h J}(1), \\
\dot{\mu}_{3 h J}(q) & =q^{2} \dot{\mu}_{3 h J}(1),
\end{aligned}
$$

where we have indicated the retention in the notation for the parameters. From (17)-(20) we see that for quota-share it is not necessary to tabulate the parameters for different retentions; it is sufficient to give the parameter values for the gross business.

By excess-of-loss reinsurance with retention $m$ we have

$$
\dot{Y}_{h J k}=\min \left(\dot{Z}_{h J k}, m\right) \text {, }
$$

and thus

$$
\dot{\alpha}_{i h J}(m)=\int_{(0, m]} z^{i} d \dot{G}_{h J}(z)+m^{i}\left[1-\dot{G}_{h J}(m)\right],
$$

where $\dot{G}_{h J}(\cdot)$ is the distribution of $\dot{Z}_{h J 1}$. In this case there is no simple connection between the parameters for the situations with and without reinsurance, and the Supervisory Service would have to provide the companies with values of the parameters for different values of $m$. For intermediate retentions the company could find the parameter values by linear interpolation. For retentions exceeding the greatest finite tabulated value, the company should use the parameter values corresponding to infinite retention (i.e., gross business). 
For surplus reinsurance we have to introduce the sum insured $\dot{S}_{h J k}$ of the $k$ th claim contained in $X_{h}$. We also introduce the claims degree

$$
\dot{R}_{h J k}=\dot{Z}_{h J k} / \dot{S}_{h J k}
$$

With retention $m$ one has

$$
\dot{Y}_{h J k}=\min \left(m, \dot{S}_{h J k}\right) \dot{R}_{h J k}
$$

It is seen that $\dot{Y}_{h J k}$ is influenced by both the distribution of the sums insured and the connection between sum insured and claim amount, and thus it is most convenient to consider both $\dot{S}_{h J k}$ and $\dot{Z}_{h J k}$ as random variables. We have

$$
\begin{aligned}
\dot{\alpha}_{i h J}(m)= & E\left(\dot{Y}_{h J_{1}}\right)^{i}=\int_{(0, m]} s^{i} \int_{0}^{\infty} r^{i} d \dot{F}_{2 h J}(r \mid s) d \dot{F}_{1 h J}(s) \\
& +m^{i} \int_{(m, \infty)} \int_{0}^{\infty} r^{i} d \dot{F}_{2 h J}(r \mid s) d \dot{F}_{1 h J}(s),
\end{aligned}
$$

where $\dot{F}_{1 h J}(\cdot)$ is the marginal distribution of $\dot{S}_{h J 1}$ and $\dot{F}_{2 h J}(\cdot \mid s)$ is the conditional distribution of $\dot{R}_{h J 1}$, given that $\dot{S}_{h J 1}=s$. If $\dot{S}_{h J 1}$ and $\dot{R}_{h J 1}$ are stochastically independent, then $(21)$ reduces to

$$
\begin{aligned}
\dot{\alpha}_{i h J}(m) & =E\left(\min \left(m, \dot{S}_{h J 1}\right)\right)^{i} E\left(\dot{R}_{h J 1}\right)^{i} \\
& =\left\{\int_{(0, m]} s^{i} d \dot{F}_{1 h J}(s)+m^{i}\left[1-\dot{F}_{1 h J}(m)\right]\right\} \int_{0}^{\infty} r^{i} d \dot{F}_{2 h J}(r) .
\end{aligned}
$$

(In many branches the claims degree is stipulated by the contract to be a number between 0 and 1 , in which case the upper limit $\infty$ could be replaced by 1 in the inner integrals in (21) and (22).) We see that, like in excess-of-loss reinsurance, there is no simple connection between the parameters for the situations with and without surplus reinsurance, and also for surplus the Supervisory Service will have to tabulate parameter values for different retentions.

The distribution $\dot{F}_{1 \mathrm{hJ}}(\cdot)$ of the sums insured depends on the composition of the portfolio and is likely to vary from company to company. It might therefore be desirable that the Supervisory Service constructs a special table of parameter values for each company, utilizing statistical knowledge about the distribution of sums of that particular company. Credibility theory may be a valuable tool in this connection. The distribution $\dot{F}_{2 h J}(\cdot \mid s)$ is to a greater extent than $\dot{F}_{1 \mathrm{hJ}}(\cdot)$ determined by technical conditions and could presumably, as an acceptable approximation, be assumed to be the same for all companies.

We have now discussed the pure unbounded versions of the reinsurance forms quota-share, excess-of-loss, and surplus. In practice, however, one often uses bounded versions and combinations of these forms. For some of these mixed reinsurance forms (e.g., combinations of quota-share and excess-of-loss) one could easily find the parameter values by the tables and formulae given above. 
By certain other forms matters are more complicated. One reinsurance form often used in practice is surplus with an excess-of-loss cover on the (surplus) retention. In this case one would need a table with two entries (surplus and excess-of-loss retentions). An alternative solution could be that the Supervisory Service on the company's request computes the parameter values for the company or provides the sufficient information for the company to compute the parameter values itself. A variant of the latter solution would be that the Supervisory Service provides the company with computer software for the computations. Then the company would only need to type in their risk volumes, reinsurance forms, and retentions.

\section{ESTIMATION OF THE MODEL PARAMETERS}

In Section 5 we consider one particular branch $h$, and for simplicity we skip the index $h$ from all quantities. We make some new assumptions.

(iv) Events referring to different years are stochastically independent.

Let $N_{j}$ denote the number of claims incurring in year $j$ and let $Z_{j k}$ denote the gross amount of the $k$ th of these claims. We also introduce a measure $p_{j}$ of the risk exposure in year $j$. We assume that $N_{j}, p_{j}$, and the $Z_{j k}$ 's are known for the years $1, \ldots, J^{*}$. In analogy to assumption (iii) we make the following assumption.

(iii') The number of claims $N_{j}$ is conditionally Poisson distributed with parameter $p_{j} \theta$, given the value $\theta$ of the unknown random parameter $\Theta_{j}$. The gross claim amounts $Z_{j k}, k=1,2, \ldots$, are i.i.d. and independent of $N_{j}$ and $\Theta_{j}$.

Note that in general $p_{j} \neq \dot{p}_{j}$, cf. the discussion in subsection $2 \mathrm{~A}$.

(v) The random parameters $\Theta_{1}, \Theta_{2}, \ldots$ are i.i.d.

Assumptions (iv) and (v) represent a slight oversimplification as they exclude the possibility of a connection between the risk conditions in neighbouring years (the road conditions around New Year will influence the results in both years).

The parameter $\Theta_{J}$ is the same in assumptions (iii') and (v) as in assumption (iii). This is perhaps a bit unrealistic as it really implies a sort of uniformity during the year of the risk conditions; as $\dot{N}_{j}$ are the claims incurred by policies being in force at the beginning of the year, the average time of incurrence of these claims would tend to be earlier than the average of the claims contained in $N_{j}$. (We are here reasoning as if failure of a company takes place at New Year. This need, of course, not be the case. On the other hand, cases of insolvency will typically be uncovered in connection with the annual solvency supervision at the beginning of the year.)

We first treat the estimation of the moments $\beta_{i}$ defined by (10). It seems reasonable to assume that all companies are influenced by the same collective risk variations such that we may pool data from all companies for the estimation of the $\beta_{i}$ 's. Thus, for the present we let $N_{j}$ be the total number of claims from all companies. 
For $\beta_{1}$ and $\beta_{2}$ we propose the unbiased estimators [cf. e.g., Sundt (1983)]

$$
\hat{\beta}_{1}=\frac{1}{\sum_{j=1}^{J^{*}} p_{j}} \sum_{j=1}^{J^{*}} N_{j}
$$

$$
\hat{\beta}_{2}=\frac{1}{\sum_{j=1}^{J^{*}} p_{j}\left(1-p_{j} / \sum_{k=1}^{J^{*}} p_{k}\right)}\left[\sum_{j=1}^{J^{*}} p_{j}\left(\frac{N_{j}}{p_{j}}-\hat{\beta}_{1}\right)^{2}-\left(J^{*}-1\right) \hat{\beta}_{1}\right] .
$$

The estimator $\hat{\beta}_{2}$ may take negative values, whereas the estimand $\beta_{2}$ is always non-negative. It is recommended that $\hat{\beta}_{2}$ is put equal to zero if the right-hand expression of (23) is negative.

We still have to estimate $\beta_{3}$. In practice one would typically use data from at most 6 years, that is, we have at most 6 realizations $\Theta_{j}$. As these realizations are not even directly observable, it seems a bit risky to perform an estimation of the third order moment without making further assumptions. We therefore add more structure to the model by assuming that the $\Theta_{j}$ 's are gamma distributed with parameters $\gamma$ and $\delta$, that is, $\Theta_{j}$ has density

$$
\frac{\delta^{\gamma}}{\Gamma(\gamma)} \theta^{\gamma-1} e^{-\delta \theta}, \quad \theta>0
$$

This gives

$$
\beta_{1}=\frac{\gamma}{\delta}, \quad \beta_{2}=\frac{\gamma}{\delta^{2}}, \quad \beta_{3}=\frac{2 \gamma}{\delta^{3}}
$$

hence

$$
\beta_{3}=2 \frac{\beta_{2}^{2}}{\beta_{1}}, \quad \kappa_{3}=2 \kappa_{2}, \quad \tau=2 \rho^{2} .
$$

We propose to estimate $\beta_{3}$ by

$$
\hat{\beta}_{3}=2 \frac{\hat{\beta}_{2}^{2}}{\hat{\beta}_{1}} \text {. }
$$

An alternative to the assumption that the $\Theta_{j}$ 's are gamma distributed, is to put $\kappa_{3}=0$. This assumption implies that $\tau=0$, and (16) reduces to

$$
E(X-E X)^{3}=\dot{\mu}_{3 J} E X+3 \dot{\mu}_{2 J} \operatorname{Var} X .
$$

We now turn to the estimation of the $\dot{\alpha}_{i j}$ 's defined by (10). In Section 4 we assumed that the values of these parameters are common to all companies. However, for some branches and reinsurance forms (e.g., surplus) it could be appropriate to assume that the parameter values vary across companies. We shall leave the question open as to whether the data are aggregate or on company level. It could also be reasonable to assume that the claim size distributions of the different companies are drawn from one and the same population. In that 
case methods from the theory of experience rating could be employed for the estimation of each single claim size distribution, but this idea will not be pursued any further here.

The assumptions made till now do not relate the available data to the $\dot{\alpha}_{1 J}$ 's, and therefore we add the following assumption.

(vi) There exist parameters $\lambda_{j}$ and $\dot{\lambda}_{J}$ such that the $Z_{j k} / \lambda_{j} ; k \geqslant 1 ; j=1, \ldots, J-1$, are i.i.d. with the same distribution as $\dot{Z}_{J 1} / \dot{\lambda}_{J}$.

Assumption (vi) may be interpreted as a way of modelling inflation, and $\lambda_{j}$ could be interpreted as a price index. The parameter $\dot{\lambda}_{J}$ might differ from $\lambda_{J}$ as the $\dot{Z}_{J k}$ 's on the average will incur earlier than the $Z_{J k}$ 's (cf. the discussion following assumption $(v))$. The $\lambda_{j}$ 's will typically vary between branches as the costs are not likely to develop equally in each branch. Assumption (vi) represents a quite rough idealization. It is not strictly fulfilled for insurance forms with fixed amount deductible (as long as the deductible is not adjusted according to the index), and it is in general also not fulfilled if the different components of the claim costs do not develop equally.

The $\lambda_{j}$ 's could be estimated either directly from the claims data or from exogeneous information on the cost level of the goods and services in question. For branches where the development in the claim amounts is caused by changes in the prices for goods and services exclusively, the $\lambda_{j}$ 's could be estimated exogeneously from price indices. This could be realistic, for instance, for civil fire insurance within the relative short spans of time in question (say 6 years), where one could assume that building technology and standard of living are approximately constant. The situation is different in branches where one in addition to a possible pure development in prices could assume that the pattern of the claims could change as a result of changes in technology and behaviour. To stick to civil fire, one might have that the standard of living changes remarkably during the observational period, that the choice of materials and way of building change, and that people start furnishing their houses more luxuriously. All such changes would be reflected in the claim amounts, even if the prices for goods and services remain constant in the building trade. A more obvious example is motor insurance, where changes in traffic regulations and traffic environments might imply that certain kinds of claims become relatively more frequent. In those branches where the development in claim amounts also to a great extent is caused by trends in technology and behaviour, one has to estimate the $\lambda_{j}$ 's directly from the available claims data.

Without loss of generality, let $\lambda_{1}=1$. It is reasonable to assume that the distribution $F$ of the $Z_{1 k}$ 's belongs to some parametric family of distributions. Appropriate classes could be log-normal, gamma, and log-gamma. If estimates $\hat{\lambda}_{j}$ of the $\lambda_{j}$ 's are found from exogeneous data, the parameters of $F$ are estimated from the ratios $Z_{j k} / \hat{\lambda}_{j}, j=1, \ldots, J^{*} ; k=1, \ldots N_{j}$, using the approximation that these ratios are i.i.d., with distribution $F$. If the $\lambda_{j}$ 's are not estimated from exogeneous data, we estimate these parameters as well as the parameters of $F$ from the observed $Z_{j k}$ 's by usual parametric methods. In most models maximum 
likelihood estimation would be preferable. Moment methods could also be applied. In the latter case $\lambda_{j}$ would be estimated by

$$
\hat{\lambda}_{j}=\frac{\bar{Z}_{j}}{\bar{Z}_{1}}
$$

with $\bar{Z}_{j}=\sum_{k=1}^{N_{j}} Z_{j k} / N_{j}$. This estimator is also Maximum Likelihood for many parametric classes. However, it will be strongly influenced by catastrophic claims, and it is therefore necessary to pay special attention to the tail properties of the model distribution.

By using the methods indicated above, we can estimate the parameters of the claim size distributions for the years $1, \ldots, J^{*}$. For the computation of the $\dot{\alpha}_{i J}$ 's, however, one also needs an estimate of $\dot{\lambda}_{J}$. In practical applications one would typically have $J=J^{*}+2$ (as the supervisory authority should have claims data from the whole of year $J^{*}$ to make the estimates that should be available for the companies at the beginning of year $J$ ). For estimation of $\dot{\lambda}_{J}$ one could use the available estimates of $\lambda_{2}, \ldots, \lambda_{J *}$ combined with available exogeneous prognoses on the development of prices.

When $\dot{\lambda}_{J}$ and the parameters of $F$ have been estimated, we obtain estimates of the $\dot{\alpha}_{i J}$ 's for excess-of-loss and quota-share reinsurance. For surplus reinsurance we also have to incorporate sums insured in the estimation, and in the following we suggest how to proceed in this case.

It is assumed that $\dot{S}_{J 1}$ and $\dot{R}_{J 1}$ are independent. Referring to formula (22), we first estimate

$$
\dot{\delta}_{i J}(m)=E\left(\min \left(m, \dot{S}_{J 1}\right)\right)^{i}
$$

and

$$
\gamma_{i J}=E\left(\dot{R}_{J 1}\right)^{i}
$$

separately and then estimate $\dot{\alpha}_{i j}(m)$ by the product of these estimates.

Let $R_{j k}$ denote the claims degree by the $k$ th claim in year $j$ (that is, $R_{j k}$ is equal to $Z_{j k}$ divided by the corresponding sum insured). It is assumed that the $R_{j k}$ 's are known for the years $j=1, \ldots, J^{*}$, and that these quantities are i.i.d. with the same distribution as $\dot{R}_{J 1}$. Then

$$
\hat{\gamma}_{i J}=\frac{1}{\sum_{j=1}^{j^{*}} N_{j}} \sum_{j=1}^{J^{*}} \sum_{k=1}^{N_{j}} R_{j k}^{i}
$$

is a natural estimator of $\gamma_{i j}$.

Let $n_{j}$ denote the number of policies in force at the end of year $j$ and $S_{j k}$ the sum insured by the $k$ th of these policies. It is assumed that $n_{j}$ and the $S_{j k}$ 's are known for the years $1, \ldots, J^{*}$. Corresponding to assumption (vi) we assume that there exist parameters $\mu_{j}$ and $\dot{\mu}_{J}$ such that the $S_{j k} / \mu_{j}$ 's are i.i.d. with the same distribution as $\dot{S}_{J 1} / \dot{\mu}_{J}$, and that the $\dot{\delta}_{i J}(m)$ 's can be estimated analogously to the estimation of the $\dot{\alpha}_{i J}$ 's for the case with excess-of-loss reinsurance. 


\section{MODIFICATIONS FOR UNSETTLED CLAIMS}

6A. In Section 4 we assumed that all claims were settled immediately when they occurred. That is, of course, a very unrealistic assumption, and we shall have to adjust for it if the system shall be of any practical applicability.

Let $u_{\min }^{\prime}$ denote the upper $\varepsilon$-fractile of the distribution of $\dot{X}_{J}$. Under assumption (ii) we have $X=\dot{X}_{J}$. Thus $u_{\min }^{\prime}=u_{\min }$, and therefore $u_{\min }^{\prime}$ can be found by the procedure outlined in Section 4. Let us now leave assumption (ii), keeping the other assumptions. Then $u_{\min }$ would typically be greater than $u_{\min }^{\prime}$. However, $u_{\text {min }}^{\prime}$ can still be found by the procedure of Section 4 .

Let us assume that the loss liability $u_{l}$ is known. An easy solution would be to require that the company should have sufficient assets to cover all payments on future claims for which the company has assumed liability at time $J$, with probability not less than $1-\varepsilon$. In addition, the company should have assets to cover $u_{l}$, that is, we should have $u \geqslant u_{\min }^{\prime}+u_{l}$. The present authors would not recommend this requirement as it neglects the stochastic nature of unsettled claims. Therefore we propose the following ad hoc procedure:

From (14) we have

$$
E \dot{X}_{h J .}=\dot{p}_{h J} \dot{\mu}_{1 h J}
$$

and as

$$
X_{h}=\dot{X}_{h J}+R_{h J}
$$

we get

$$
E X_{h}=\dot{p}_{h J} \dot{\mu}_{1 h J}+u_{h l}
$$

(the meaning of the new symbols introduced should be obvious). The ad-hockery consists in inserting $E X_{h}$ given by (24) into (15) and (16) to find expressions for $\operatorname{Var} X_{h}$ and $E\left(X_{h}-E X_{h}\right)^{3}$. Then these moments are inserted into (7)-(9) to find the moments of $X$, and finally $u_{\min }$ is found by insertion in (6).

6B. For the parameter estimation in Section 5 we assumed that the $N_{h j}$ 's and the $Z_{h j k}$ 's were known. However, as in practice there will still be unreported and unsettled claims, $N_{h j}$ has to be estimated by the number of reported claims plus the estimated number of unreported claims, and $Z_{h j k}$ by paid amount plus reserved amount.

\section{FURTHER DEVELOPMENT}

7A. Sections 4-6 outlined a provisional procedure for the computation of $u_{\text {min }}$. As should be clear from the preceding, much work has to be done before this procedure could be put into practice. Data will have to be collected from the companies. For the purpose of estimating the $\alpha$ 's, one has to find reasonable parametric classes of distributions that can be fitted to the claim amounts. One should find reasonable measures of risk exposure. As a provisional solution, the Working Party suggested to use the premium as a measure of risk volume, but 
as pointed out in Section 3, this is by no means an optimal solution, and the search for more reasonable measures should be given high priority.

In the following we give some suggestions for further development of models and methods.

7B. In Section 6 it was assumed that the loss liability $u_{h l}$ is known for each branch $h$, and no guidelines were offered as to how to find this quantity. As a provisional solution, the Working Party suggested that one should just use the company's actual loss reserve without proposing any specific method for assessing this reserve. This is of course a most unsatisfactory solution, and it is important to find standardized, objective methods according to which $u_{h l}$ should be calculated.

It has been argued that in many branches the companies use subjective individual case estimation, incorporating all the knowledge and intuition of experienced claim-handlers, that is, information not being incorporated in objective methods, and thus the actual loss reserve is the best possible estimate of $u_{h \text { l }}$. For internal purposes in the companies this argument seems very reasonable, but for supervisory purposes it is not satisfactory. With such a system the company would have too great freedom to determine the size of the loss liability and, thereby, of the minimum reserve.

For the development of models and methods for the estimation of $\boldsymbol{u}_{h l}$ the Supervisory Service will have to collect run-off data from the companies.

As already indicated, even if good estimates for the $u_{h l}$ 's are available, the procedure outlined in subsection $6 \mathrm{~A}$ is most ad hoc, and one should principally incorporate the mechanism of claims settlement into the model. However, this seems to be a very complicated matter, and until a reasonable solution is found, we suggest to use the procedure of Section 6 as a preliminary solution.

7C. Some branches consist of many small and relatively equal risks. For such branches the provisional model could be fairly realistic. In other branches there could be great differences between the single risks. The total risk could be dominated by a few great single risks, and for such branches the provisional model is not appropriate. There is a need for development of special methods for consideration of great risks. Also for branches like credit insurance and received reinsurance the model assumptions do not seem to be very realistic, and more appropriate models ought to be worked out for these branches.

\section{SOME NUMERICAL ILLUSTRATIONS}

In the pilot study performed by the Supervisory Service, risk statistics were collected in a number of insurance branches from six major Norwegian non-life insurance companies, and the model in Section 4 was fitted to the data in each separate branch. Claim amount distributions were simply estimated by the observed empirical distributions.

In the table overleaf we report on some results thus obtained for the branches $\mathrm{CP}=$ civil property, $\mathrm{ML}=$ motor liability, and $\mathrm{MO}=$ motor "others". For a number of alternative specifications of size and composition of the portfolio and 


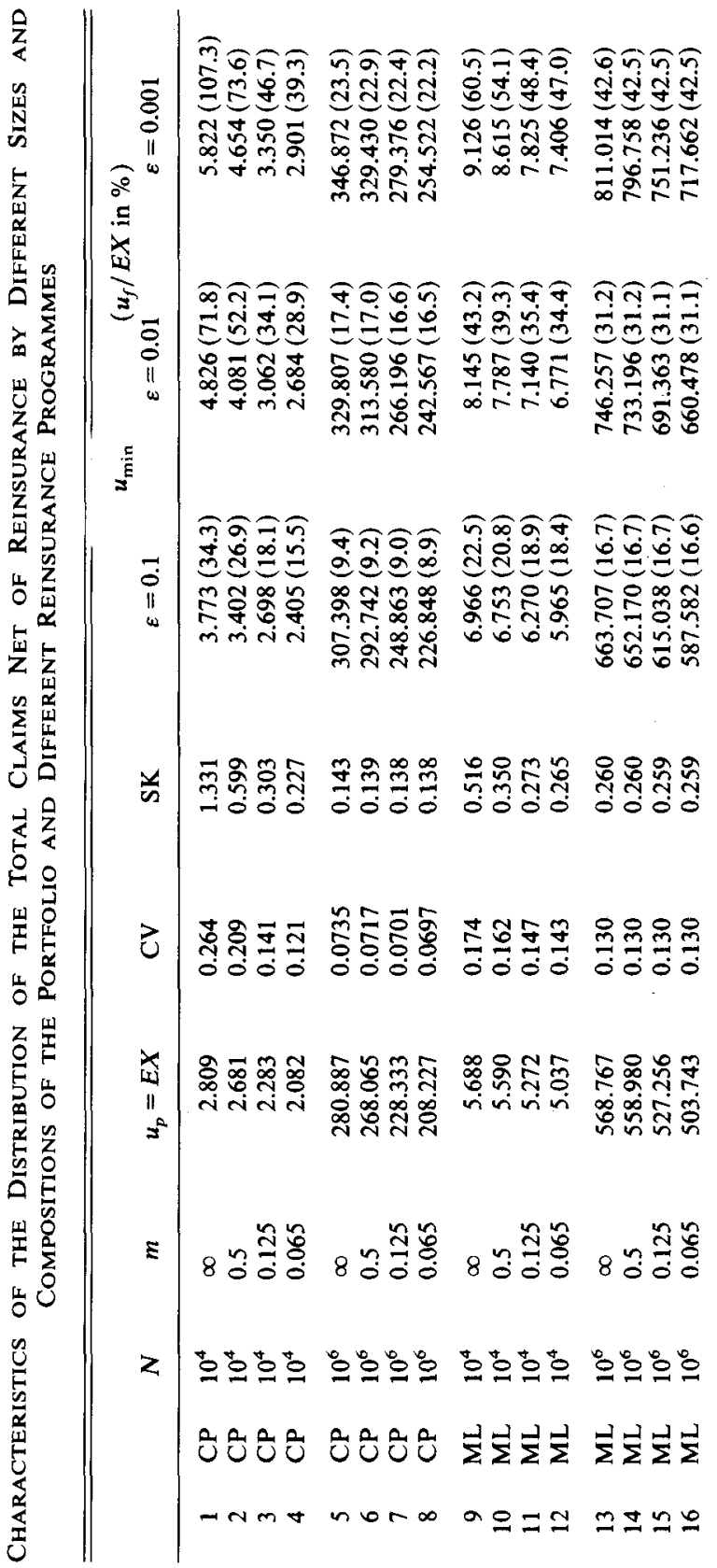




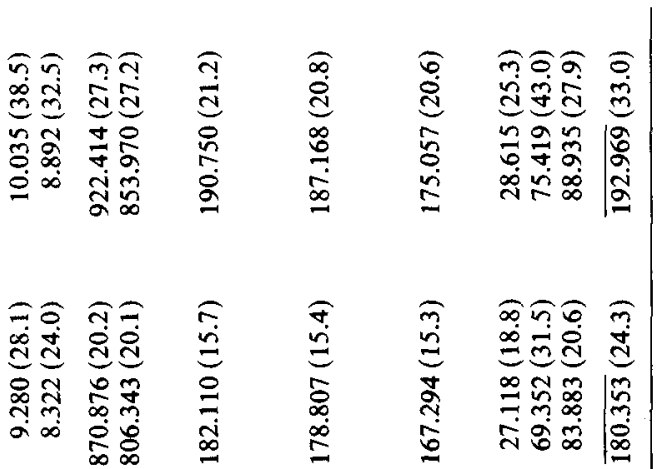

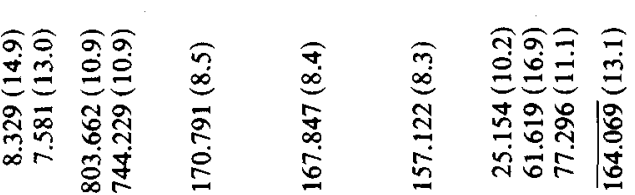

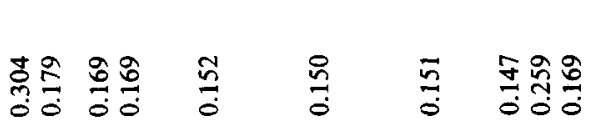

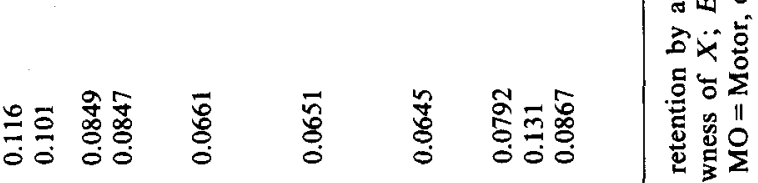

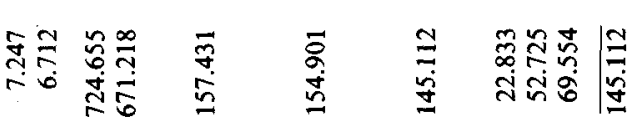

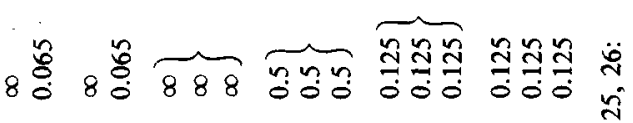

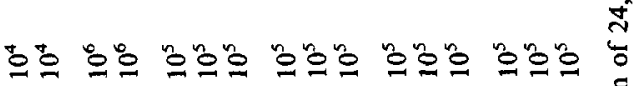

울울율

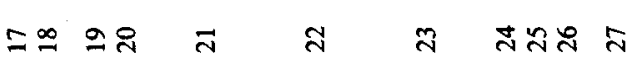

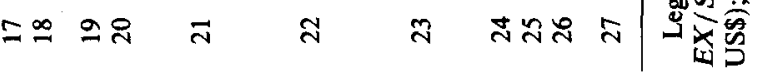


levels of net retention the table shows expected value, coefficient of variation, skewness, and upper $10 \%-, 1 \%-$, and $0.1 \%$-fractiles of the distribution of $X$. The number in parentheses is the fraction $u_{f} / E X$, which can be interpreted as a security loading. The fractiles have been calculated by use of the NP-approximation (6).

The results in the table are only intended as an illustration. The quality of the data was poor, and instead of fitting the model to these, we could simply have specified some various sets of values of the parameters considered to be representative for the major non-life branches. We shall comment briefly on the results.

A comparison of rows $1,9,17$ (or $5,13,19$ ) with respect to coefficient of variation, skewness, and fractiles shows that in the case of no reinsurance $\mathrm{CP}$ has the heaviest tail of the three branches considered, and MO has the lightest tail. This reflects the circumstance that fire claims are rare and large, whereas MO-damages are frequent and comparatively small.

This pattern is distorted by introduction of reinsurance, as can be seen by comparison of e.g., rows 4,12 , and 18 . The explanation is that $C P$ is less sensitive to fluctuation in collective risk factors (e.g., weather) than are ML and MO. An excess-of-loss cover effectively reduces the impact of large claims whereas it cannot eliminate the influence of random fluctuations of the claim frequency.

The effect of reinsurance can be further investigated by examination of rows corresponding to a fixed branch and fixed exposure $p$. By comparison of rows 1-4, which refer to a CP-portfolio comprising only 10000 policies, it is seen that reinsurance is an effective risk-reducing measure for a small, heavy-tailed business. The perhaps best indication are the "loadings" given in parentheses. If we look at rows 5-8 referring to a large portfolio in the same branch, we find that reinsurance is almost without any effect. The size of the business has practically annihilated the pure compound Poisson variation, and the only variation remaining is that of the claim frequency parameter, which, as explained above, is not damped by excess-of-loss arrangements.

Rows 21-23 refer to a "typical" medium-sized Norwegian company with 100000 risks insured in each of the three branches considered. The effect of excess-of-loss reinsurance is negligible as measured by the shape parameters of the distribution and by $u_{f} / E X$, that is to say excess-of-loss behaves practically like quota-share.

Comparison of row 23 to rows $24-26$ and 27 opens another aspect: By pooling the three single-branch businesses into one company, the required provisions for fluctuation reserves are substantially reduced. Moreover, a comparison of row 21 to each of the rows 5,13 , and 19 shows that the risk is more efficiently balanced by pooling of different branches than by growth of business in one and the same branch. The explanation is, of course, that the effect of collective random risk factors cannot be reduced by growth in one single branch. (In passing we mention that the results in different branches are assumed to be independent. In practice this is not the case with ML and MO since they are influenced by the same collective risk factors.) 
Finally, a solvency control application: Suppose the company represented in rows 21-23 have only 168 mill. NOK in secure assets. Then, with $\varepsilon=0.01$ in the solvency requirement (1), a net retention of 0.125 mill. NOK in all branches can be approved by the Supervisory Service, whereas a net retention of 0.5 mill. NOK cannot.

\section{REFERENCES}

Beard, R. E., Pentikäinen, T. and Pesonen, E. (1984) Risk Theory, 3rd ed. Chapman \& Hall Ltd., London.

HARLEM; G. et al. (1983) Forsikring i Norge. NOU 1983:52. Universitetsforlaget, Oslo.

JOHNSEN, T. et al. (1984) Solvenskontroll av skadeforsikringsselskaper. Norwegian Insurance Supervisory Service, Oslo.

RANTALA, J. (1982) Solvency of Insurers and Equalization Reserves, Vol. II. Insurance Publishing Company, Helsinki.

SUNDT, B. (1983) Parameter estimation in some credibility models. Scandinavian Actuarial Journal $239-255$. 